\title{
An Uncommon Experience During Removal of a Foreign Body from the Ear of a Child
}

\author{
Sanjoy Kumar Ghosh, ${ }^{1}$ Abir Chaudhury ${ }^{1}$
}

ABSTRACT

An oblate spheroid bead was removed from the middle ear through post-auricular approach by threading the foreign body with polypropylene suture material.

$\underline{\text { Keywords }}$

Foreign Bodies; Ear, Middle; Child; Polypropylene.

$\Delta$ two year old male child was presented to our medical college hospital on August 19, 2015 with the history of accidental introduction of foreign body in right ear. According to his mother the foreign body was a bead of her necklace. The bead was spherical in shape but flattened on two sides like the shape of our Earth. Geometrically we can say it was oblate spheroid or oblate ellipsoid in shape. The child was taken to a nearby medical facility, where doctors tried to remove the foreign body by aural syringing initially and then with the help of microscope through an endaural approach. The foreign body could not be removed as it was deep inside the ear canal and the patient was referred to tertiary health care centre.

Foreign bodies in the ear canal are very common. We generally remove a solid foreign body from ear by aural syringing especially when the foreign body resided lateral to the isthmus of external ear canal. A foreign body residing medial to the isthmus of external auditory canal can be removed by microsurgical instruments, especially if the foreign body is spherical in shape.

Here, in this case when we examined the patient's ear with aural speculum we could not appreciate any foreign body because of blood clots occluded the ear canal mixed with discharge. So we admitted the child and started conservative management for 5 days. The ear was examined under general anaesthesia with the help of an operating microscope. The tympanic membrane was found ruptured and top of foreign body could be seen through the perforation with great difficulty (as the ear canal of the child was very narrow and oedematous). So, we first made a post-aural incision from the highest attachment of the pinna following the curve of the retroauricular groove $1 \mathrm{~cm}$ behind it slanting posteriorly (as in tympanoplasty).

After elevating the tympanomeatal flap, we found that the foreign body was in the middle ear cavity medial to bony annulus within the hypotympanum and inferior to handle of malleus. We first used micro cup forceps and alligator forceps but with these forceps we could not grasp the ellipsoid shaped bead. Then we tried a curved needle by introducing the tip of the needle to one the openings that were present at both pole of the bead. But what we experienced that when we tried to pull the foreign body away from handle of malleus, tendency of the foreign body was to rotate it's another surface and slips into postero-inferior portion of hypo tympanum and used to stick against the bony annulus. We repeatedly tried with the same instrument but all attempts went in vain. Then we had left two option either we had to drill the bony annulus or we had to sacrifice the ossicular chain to remove the foreign body. But we didn't want to drill out the annulus as it may distort normal anatomy

1 - Department of ENT, Medical College, Kolkata

Corresponding author:

Dr Sanjoy Kumar Ghosh

email: sanjoyghoshcmc007@gmail.com 


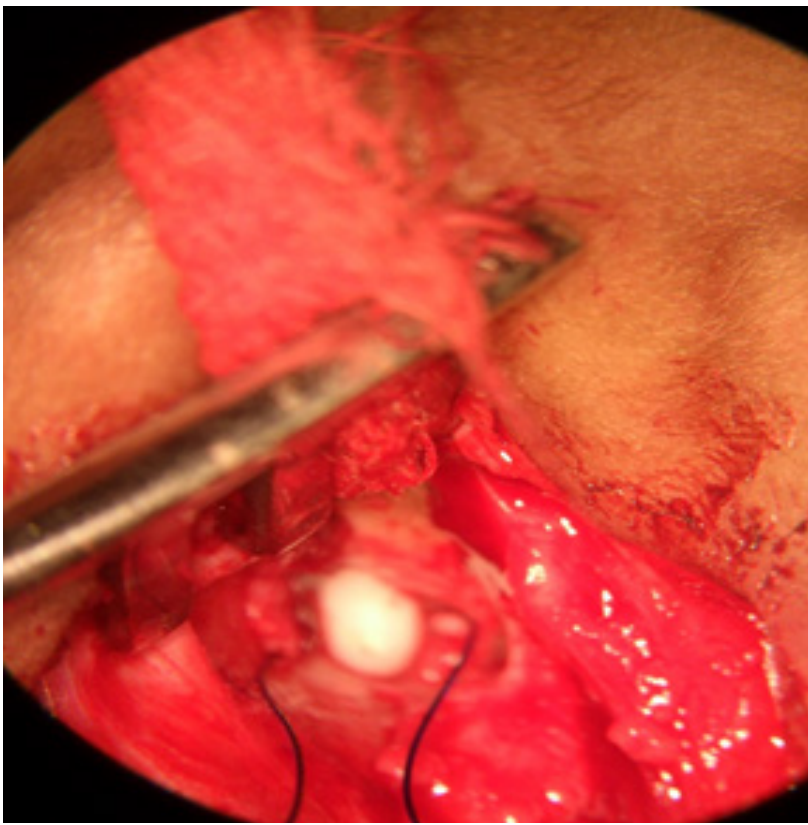

Fig. 1 The bead is being extracted by pulling the polypropylene thread

causing post-operative blunting of tympanic membrane.

Then, we all appreciated the fact that if the bead could be positioned so as to equator of the oblate spheroid foreign body looked directly towards outside we could easily remove the foreign body. But we didn't have such forceps to hold the foreign body in aforesaid position and remove it. We got an unique idea from our fellow surgeon that if we would be able to pass a Prolene $\AA$ thread into the hole passing through the centre of the bead and then remove it from other side of the bead, we could easily solve the problem. We took a 4-0 Prolene $\AA$ thread, held it with an alligator forceps and passed it through the centre hole and grasped it from other side as it is seen in the photograph. Then a gentle pull of the thread was all we needed to do (Fig.1). The foreign body came out very easily from middle ear cavity without forcing handle of malleus. Post-operative ossicular chain was completely intact. We packed middle ear cavity with gel foam.

After 3 weeks follow up, patient's tympanic membrane was healed with features of OME and after 6 weeks follow up, effusion disappered, there was no blunting and cone of light came back, moreover, the patient was very happy without complaining of impairment of hearing. From that incident what we got lesson is that we can always modify surgical steps with judicious thinking keeping the surgical principal intact to achieve a better goal in long time. 